\title{
Low Cost Optical-Electronic Sensor Development Based on Raman Spectroscopy for Liquid
}

\author{
Lukman Aji Kusumo \\ Department of Electrical Engineering \\ Institut Teknologi Sepuluh Nopember \\ Surabaya, Indonesia \\ lukman.18071@mhs.its.ac.id
}

\author{
Totok Mujiono \\ Department of Electrical Engineering \\ Institut Teknologi Sepuluh Nopember \\ Surabaya, Indonesia \\ totok.m@ee.its.ac.id
}

\author{
Hendra Kusuma \\ Department of Electrical Engineering \\ Institut Teknologi Sepuluh Nopember \\ Surabaya, Indonesia \\ hendraksm@ee.its.ac.id
}

\begin{abstract}
Spectroscopy is a method that used to identify chemical structure of substances using its spectral pattern characteristics. Optical spectroscopy term can be applied to any kind of optical photon interactions with matter. Raman spectroscopy essentially shows spectral response like the wavelength of scattered light is shifted regarding initializing excitation wavelength. In this paper, we propose a design of low cost optical-electronic sensor based on Raman spectroscopy. This low cost optical-electronic sensor employs a violet-blue $405 \mathrm{~nm}$ wavelength laser diode, a biconvex lens with $5 \mathrm{~cm}$ diameter and focus point, a test tube, and a Complementary Metal Oxide Semiconductor (CMOS) sensor. We tested this low cost optical-electronic sensor based on Raman spectroscopy in dark condition. Combination of these hardware and components can provide measurement result to any liquid sample. From this experiment, even all liquid samples that used to test this combination of hardware and components are transparent, they still have different Raman spectra. This combination of hardware and components can be implemented into some application for instance body liquid measurement such as blood. We only use five wavelengths start from $500 \mathrm{~nm}, 550 \mathrm{~nm}, 570 \mathrm{~nm}, 600 \mathrm{~nm}$, and $650 \mathrm{~nm}$. This experiment result denotes every liquid samples that has same transparent visual properties such as tap water, refill drinking water, mineral water, $70 \%$ isopropyl alcohol, 95\% isopropyl alcohol, mixture of $70 \%$ isopropyl alcohol and single drop of iodopovidone, cloth lubricant, and dimethyl sulfoxide (DMSO), still can be distinguished by its spectra.
\end{abstract}

Keywords-CMOS sensor, laser diode, Raman scattering, Raman spectroscopy, spectroscopy

\section{INTRODUCTION}

Spectroscopy is a method that widely used for identifying chemical structure of substances using its spectral pattern characteristics (fingerprinting). There are wide variety of spectroscopy techniques and all of them provide solutions to some interesting and challenging analytical problem. Previously, Raman scattering is less used because of the problem with sample degradation and fluorescence. Recent developments in instrument technology such as laser technology have streamlined the equipment and diminishes the problem significantly [1].

Optical spectroscopy term can be attached to any type of optical photon interactions with matter [2]. Laser Raman spectroscopy is based on the Raman effect, a spectroscopy technique that analyzed the scattered light spectrum by directly measure Raman scattered light generated by sample due to laser irradiation [3]. It is a non-destructive test and a safe way to sustainable analytics since it does not require chemical reaction [4]. Raman spectroscopy is a noninvasive and sensitive optical analysis technology based on inelastic scattering. It also provides the conformation structure of molecules and specific fingerprint-type information [5]. The advantages of Raman spectroscopy are little sample preparation, convenient operation, less time consuming, and high accuracy [6].

Raman spectroscopy deals with the interaction between light and matter when the light is scattered. The electrons in an atom may be excited to virtual and excited states [7]. For many years, Raman spectroscopy has been a powerful tool for study of molecular vibration and rotations. In the pre-laser era, however, its main drawback was a lack of sufficiently intense radiation sources. The introduction of laser has indeed revolutionized this classical field of spectroscopy. The research activities in laser Raman spectroscopy have recently shown an impressive expansion and a vast literature on this field is available. Raman spectroscopy has been extensively used in many areas such as physics, molecular biology, atmosphere research, and medicine. Its theoretical development has surfaced in every field of application to specific needs and requirements [8].

In this paper, we propose a design of low-cost opticalelectronic sensor based on Raman scattering using violetblue $405 \mathrm{~nm}$ laser diode as a light source, biconvex lens as collecting lens, and CMOS (Complementary Metal Oxide Semiconductor) photodetector arrays as detector. Violetblue laser (405nm wavelength) has advantages over their longer wavelength since it is possible to have optimal excitation for high emission and wider spectral of visible light [9]. Adafruit AS7262 CMOS sensor has been used as detector. It has six wavelength channels to respond the excitation. CMOS Sensor is smaller in size and consume less power [10].

\section{THEORITICAL BACKGROUND}

Light emits from sources with various physical embodiments [11]. Under most conditions, light acts like a wave. Light is composed of oscillating electric and magnetic fields at a certain constant velocity. The electric fields and magnetic fields are perpendicular to each other and both perpendicular to the direction of travel. The magnetic and electric field also have specific direction and magnitude [12]. A light ray usually experiences scattering when it encounters any kind of inhomogeneities of the medium in its path [13].

When interacting with a sample, incident light of intensity $I_{0}$ may be partly reflected at optical interfaces $\left(I_{R}\right)$, it may be scattered $\left(I_{S}\right)$ and competing with fluorescence $\left(I_{F}\right)$, absorbed in the sample $\left(I_{A}\right)$, and the rest of part will be transmitted $\left(I_{T}\right)$. According to the law of conservation energy, energy balance of incident light may be written as,

$$
I_{0}=I_{A}+I_{T}+I_{R}+I_{S}
$$




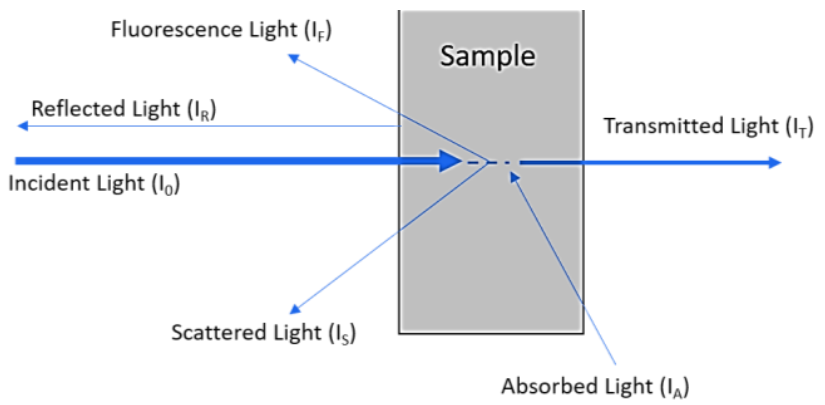

Fig. 1. Energy balance of incident light upon interaction with a sample

The light intensities $I_{0}, I_{T}, I_{R}$, and $I_{S}$ can effortlessly be measured by putting a detector at the corresponding position. All chemical information about sample goes into $I_{A}$, but this value cannot be evaluated directly. $I_{A}$ can only be accessed by assessing Eq. (1). In all commercial spectrometers, only one detector is used to measure a couple of intensity values. It is the goal of sample preparation to bring the remaining intensities to zero or at least very close to it. Fluorescence light $\left(I_{F}\right)$ itself is not a light produced by scattering process because fluorescence is not a scattering process. Fluorescence emission from most liquid and solid does not have the vibrational fine structure that appear on scattering. Even weak fluorescence light can be much stronger than scattered light, overwhelming the weak scattered light [14][18].

The Raman effect is weaker equivalent of Rayleigh scattering, and usually observed at higher intensities. On Rayleigh scattering, each scattering event requires collaborative process of photon absorption and emission, without the need of matching energy level between absorbed and emission photon. As the result of this process, the scattered light undergoes an overall transition from one energy level to another, known as Stokes line and antiStokes line [15].

Stokes Raman scattering occurs from a transition that starts at the ground state of vibrational energy level and finishes at higher state of vibrational energy level, whereas anti-Stokes Raman scattering involves a transition from higher to lower vibrational energy level. At ambient temperatures, most molecular vibrations are in ground state, so the anti-Stokes transition are less likely occur than Stokes transition [16]. The Raman effect can be excited in the UV region, the visible region, or in the NIR region [17].

One of example for Raman spectrum is Raman spectrum of chloroform, as shown in "Fig. 2.". This chloroform sample measured with Raman spectroscopy method using excitation light with $514.5 \mathrm{~nm}$ wavelength. A Raman spectrum consists of scattered intensity plotted vs energy. Each peak corresponds to a given Raman shift from incident light energy. The Stokes and anti-Stokes Raman peaks are symmetrically positioned about the Rayleigh peak, but their intensities are not same except for low vibrational energies. The $\mathrm{x}$ axis is shown in three different scales but is normally plotted as Raman shift in reciprocal centimeters relative to the laser frequency. Although the Stokes Raman to the right is negative frequency shift, convention assign Stokes Raman shifts as positive number [18].

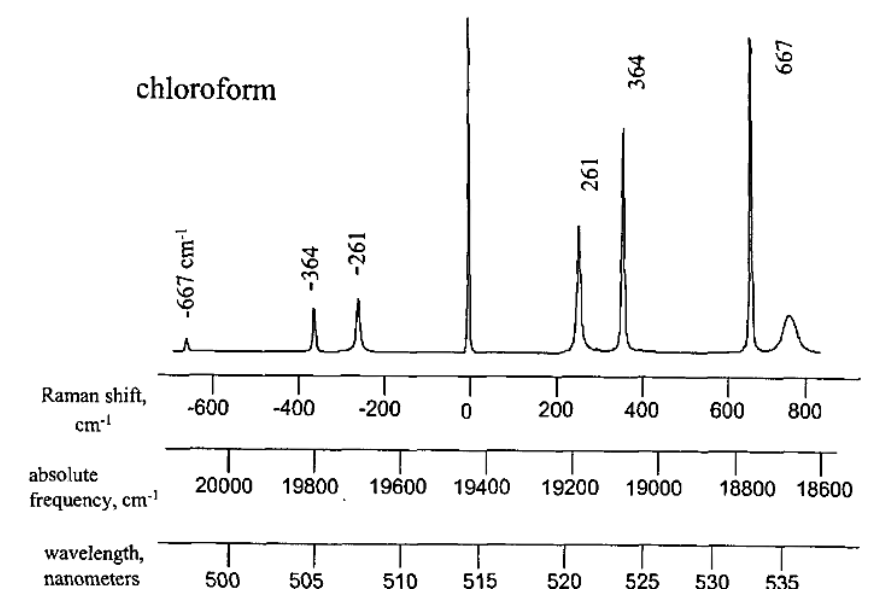

Fig. 2. Raman spectrum of chloroform [18]

Laser radiation involves an amplification process of photons. Main advantage of the lasers is the narrow wavelength band and high spectrum resolution. Semiconductor lasers are compact and efficient and can emit up to $100 \mathrm{~W}$ in $\mathrm{CW}$ (continuous wave) mode. They are successfully replacing traditional pumping sources such as arc lamps [2][19]. "Fig. 3." Shows typical emission spectrum characteristic from a $405 \mathrm{~nm} 100 \mathrm{~mW}$ laser diode. Even it has narrow wavelength band, $405 \mathrm{~nm}$ laser wavelength still spans out from around 390nm wavelength until around $420 \mathrm{~nm}$ wavelength. The $405 \mathrm{~nm}$ wavelength is only around on its peak.

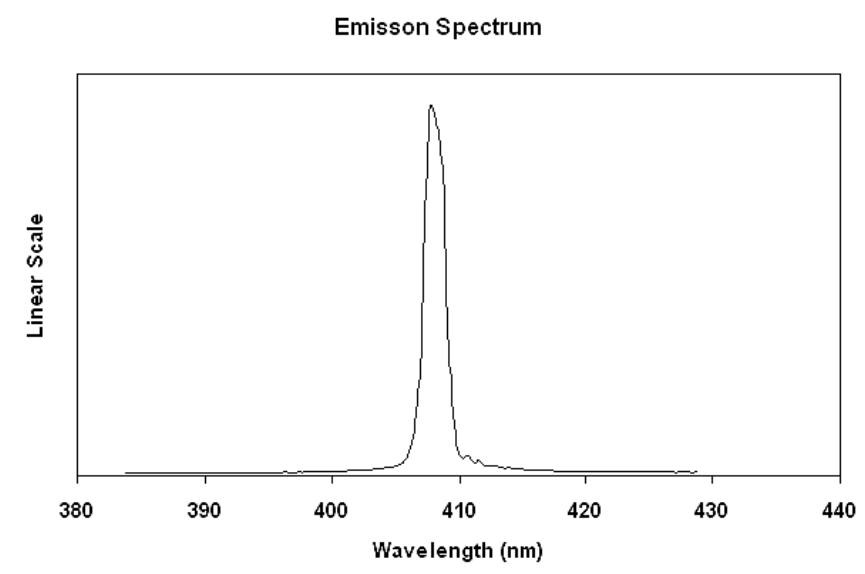

Fig. 3. Typical emission spectrum characteristic from $405 \mathrm{~nm} 100 \mathrm{~mW}$ laser diode [21]

CMOS sensor is a type of active pixel sensor made using CMOS semiconductor process [20]. CMOS sensor consists of photo-sensing element and a switching MOSFET in each individual pixel. CMOS with type of active pixel sensor today presents performance close to or better than their CCD equivalent [21].

AS7262 is a cost-effective multi-spectral sensor-onchip solution designed to address spectral ID application. This highly integrated devices deliver six-channel multi spectral sensing in the visible-wavelength from $430 \mathrm{~nm}$ to 
$670 \mathrm{~nm}$ with full-width half-max (FWHM) of 40nm as shown by "Fig. 4.".

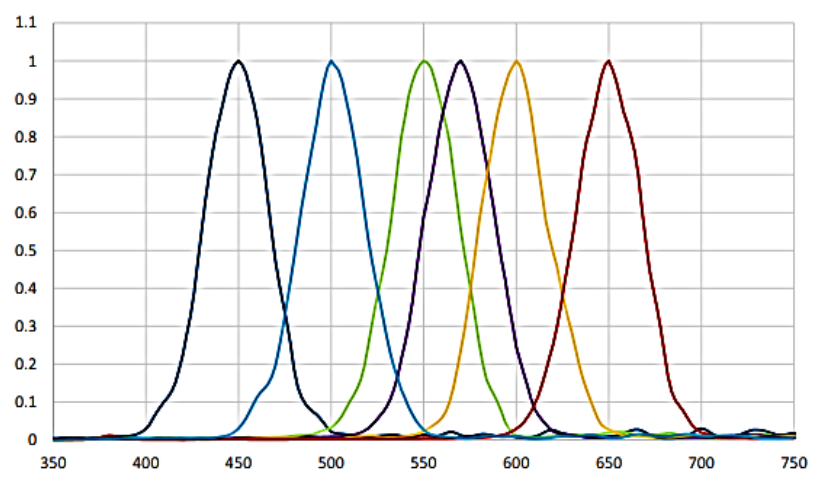

Fig. 4. Spectral response of AS7262 six-channel spectral sensor [23]

This sensor also integrates gaussian filter into standard CMOS silicon. It is compact 6-channel spectrometry solution with minimal measurement drift over time or temperature, with robust package and include built-in aperture, also equipped with electronic shutter control or synchronization, can operated with low voltage and without additional signal conditioning required [22].

\section{INSTRUMENTATION DEVELOPMENT}

The developed instrumentation consists of laser, test tube, collecting lens, detector, microcontroller, and laptop.

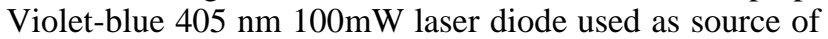
monochrome light. We use that laser specification because it has short wavelength. Since Raman scattering shifting away the scattered light wavelength from its excitation wavelength, we can acquire the scattered light in the visible light wavelength. This laser, with its $100 \mathrm{~mW}$ power, is powerful enough to penetrate some liquid sample that a little bit difficult for light to pass. Collecting lens consists of a biconvex lens with diameter of $5 \mathrm{~cm}$ and has $5 \mathrm{~cm}$ of focal point. This collecting lens is made from glass. The purpose of collecting lens is to collect and refocus the scattered light from the test tube filled with liquid samples that irradiated by laser into the detector.

We also use Adafruit AS7262, six channel visible color spectrum CMOS sensor breakout board as detector. CMOS sensor is preferred here because it is widely available, small, and consume less power. AS7262 by default uses I2C interface on the chip to communicate with microcontroller and can give output 16-bit raw values. This detector has six integrated visible light sensing, realized by silicon interference filter at $450 \mathrm{~nm}, 500 \mathrm{~nm}, 550 \mathrm{~nm}, 570$ $\mathrm{nm}, 600 \mathrm{~nm}$, and $650 \mathrm{~nm}$ channel.

Microcontroller that we use are an Arduino Nano attached to an I/O extender add-on board. We use that microcontroller because it has a lot of supports, widely available, and affordable. The function of this microcontroller in the developed instrumentation is reading data from detector that receive scattered light and then convert that data into raw measurement number. Laptop used to connect microcontroller and processing raw data that received by microcontroller from detector. The data processing that performed by this laptop are normalization for raw data received from detector and data plotting for normalized measurement data. Illustration for this developed instrumentation shown by "Fig. 5."

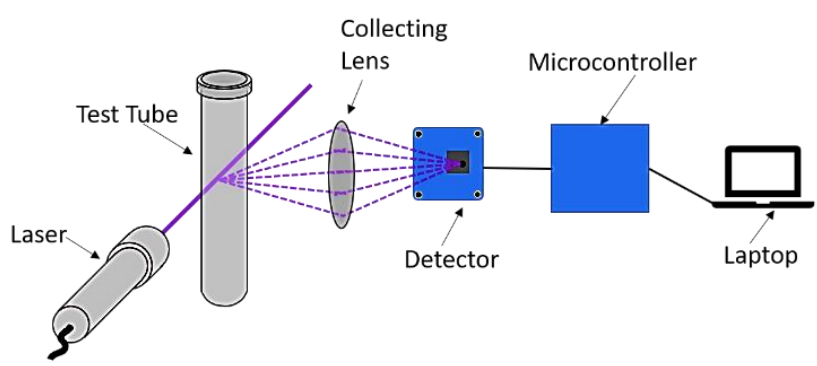

Fig. 5. Illustration of developed instrumentation for Raman spectroscopy measurement

Then, illustration of the developed instrumentation that showed in "Fig. 5." realized by designing 3D model of the corresponding support part such as holder for each component and case. Realization of the developed instrumentation is shown by "Fig. 6.". In "Fig. 6.", there are five structure that very important in this developed instrumentation such as laser holder, test tube holder, collecting lens holder, detector holder, and the case. The laser holder is used to house the $405 \mathrm{~nm} 100 \mathrm{~mW}$ laser that we use as excitation source. The test tube holder is there to hold the test tube filled with liquid sample. The collecting lens holder is used to hold the collecting lens in place.

Behind the collecting lens holder, there is detector holder. This detector holder holds the detector in place. This detector holder equipped with mounting hole to mount and fasten the detector with bolt and nut. Both of them also featuring adjustable holder, so we can adjust the height of the collecting lens and detector based on scattered lights position. To lock the position, we use small locking pins that hold the desired position. This small pin locks also modelled with $3 \mathrm{D}$ design software and printed with $3 \mathrm{D}$ printer.

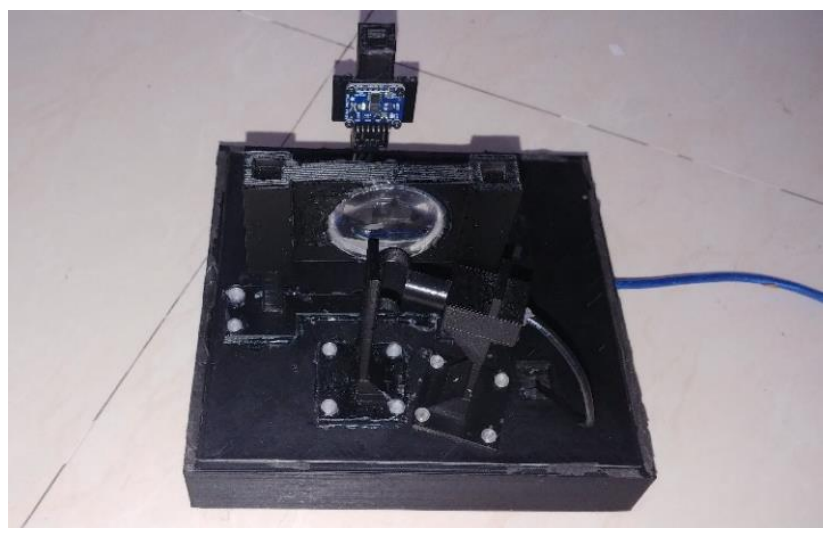

Fig. 6. Realization of developed instrumentation for Raman spectroscopy measurement

Entire set of holders are fastened on the base with locking pins like the one that used on collecting lens holder and detector holder, but larger and slightly different design. This larger pin locks designed specifically for this developed instrumentation to press fit the holder to the base that also functioning as the lid of the case. The large pin 
locks also modelled with 3D design software and printed with 3D printer. The case provides housing for microcontroller. This microcontroller can be accessed from the top of the case by lifting the lid of the case.

To do the Raman spectroscopy, we must do it in dark environment. It is intended to reduce any background color spectra that can be detected by detector, so the detector can intensively measure light spectra from Raman spectra produced by test tube and liquid sample inside the test tube and not influenced with background color spectra. To accomplish this, we made a box from cardboard with opening side at bottom to close entire experiment setup.

That cardboard box painted with black paint inside and outside to simulate dark environment and cancel out the light from outside that enter the cardboard box. After we close entire experiment setup with the "dark" box, the violet-blue laser turned on. The violet-blue laser emits violet-blue light (405 $\mathrm{nm}$ wavelength) that enter the test tube filled with liquid sample that we test. The violet-blue light from laser then scattered by test tube and liquid sample inside the test tube.

The scattered light from test tube filled by liquid sample collected by collecting lens and refocused to the detector. The scattered light that detector receives processed with microcontroller that connected to a laptop and outputted as raw measurement number. Then the laptop will be collected all the raw measurement number to form a raw measurement data and do further data processing like normalization and graphing the normalized measurement data.

For liquid samples, we mainly use transparent liquid as liquid samples. We use $3 \mathrm{ml}$ of tap water, $3 \mathrm{ml}$ of refill drinking water, $3 \mathrm{ml}$ of mineral water, $3 \mathrm{ml}$ of $70 \%$ isopropyl alcohol, $3 \mathrm{ml}$ of $95 \%$ isopropyl alcohol, mixture of $70 \%$ isopropyl alcohol with single Iodopovidone droplet with total volume of $4 \mathrm{ml}$ then picked with measuring pipette of $3 \mathrm{ml}, 3 \mathrm{ml}$ of cloth lubricant, and $3 \mathrm{ml}$ of dimethyl sulfoxide (DMSO). We also measure condition without test tube and when the test tube is still empty using Raman spectroscopy technique.

\section{MEASUREMENT PROCESS AND DISCUSSION}

Before we do the measurement, the liquid sample picked from sample container and then pour down into a 3 $\mathrm{ml}$ test tube with measuring pipette. Then, the test tube secured into its holder. A $405 \mathrm{~nm}$ laser diode placed next to the test tube. This laser placed as close as possible to the test tube to eliminate reflection that occur if there any space between the test tube and the laser. Then the liquid sample tested by turning on the laser and then detector detects the scattered light formed from excitation light scattered by liquid sample inside the test tube that collected and refocused by collecting lens. This test conducted with black-painted cardboard box covers entire developed instrumentation, so there is no external light interfere with the measurement result.

After we measured Raman scattering as spectroscopy method with every liquid samples and test in some specific condition such as the condition without the test tube and the condition with empty test tube to test the developed instrumentation, we obtained the raw measurement data of Raman scattering as spectroscopy technique. The raw measurement data are the data that consist of unrefined number in the form of 16-bit raw data that come directly from six received light spectra by CMOS sensor, but only five channels were used.

We normalized the raw measurement data from Raman spectroscopy to rescale data value into 0 and 1 range with the maximum raw value of the detector. The maximum raw value of the detector is 51,201 because this detector reads the input from all channel into 16-bit raw data with unsigned integer data type. This means that the value that can be measured up to 65,536 but the detector only able to detect up to 51,201 so the maximum value is 51,201 and because the data type is unsigned integer, it only use positive value span from 0 to 51,201. That maximum value subtracted with all the detected value on each wavelength to create the normalized measurement data. All normalized measurement data from Raman spectroscopy then visualized in form of graph for clear depiction of what happen when we do Raman spectroscopy technique to every liquid samples and some other specific conditions. Raman spectroscopy normalized measurement data graph are shown by "Fig. 7".

When we test developed instrument without test tube and with empty test tube, we found that light spectrum received by the detector is very small when we did not place test tube in developed instrument, but we also notice very high yellow color spectrum when we placed empty test tube. These events are shown by "Fig. 7. a." and "Fig. 7. b.". In "Fig. 7. a.", this event is caused by laser light is not scattered enough toward the detector. "Fig. 7. b." shows light excited from $405 \mathrm{~nm}$ laser only scattered on the test tube without scattered again by liquid sample.

Tap water consists of water $\left(\mathrm{H}_{2} \mathrm{O}\right)$ with some mineral like calcium $\left(\mathrm{Ca}^{2+}\right)$, magnesium $\left(\mathrm{Mg}^{2+}\right)$, sodium $\left(\mathrm{Na}^{+}\right)$and also some contaminant like chlorine $\left(\mathrm{Cl}^{-}\right)$, nitrate $\left(\mathrm{NO}^{3-}\right)$, and sulfate $\left(\mathrm{SO}_{4}^{2-}\right)$. Mineral water and refill drinking water have same material composition such as water $\left(\mathrm{H}^{2} \mathrm{O}\right)$, calcium $\left(\mathrm{Ca}^{2+}\right)$, magnesium $\left(\mathrm{Mg}^{2+}\right)$, potassium $\left(\mathrm{K}^{+}\right)$, and sodium $\left(\mathrm{Na}^{+}\right)$. The difference between mineral water, tap water, and refill drinking water was the possibility of added contaminants. Mineral water is likely less contaminated with contaminant than tap water and refill drinking water. The spectra of the tap water, refill drinking water, and mineral water are shown by "Fig. 7. c.", "Fig. 7. d.", and "Fig. 7. e." respectively. Between tap water, refill drinking water, and mineral water as liquid sample, mineral water has highest yellow color spectrum when we compared to tap water and refill drinking water.

$70 \%$ and $95 \%$ isopropyl alcohol have same chemical formula, $\mathrm{C}_{3} \mathrm{H}_{8} \mathrm{O}$. They also visually looking same. They only differ on its purified water concentration. 70\% isopropyl alcohol has $30 \%$ purified water concentration, meanwhile $95 \%$ isopropyl alcohol only has $5 \%$ purified water concentration. DMSO (dimethyl sulfoxide) has chemical formula $\mathrm{C}_{2} \mathrm{H}_{6} \mathrm{OS}$. The spectra of $70 \%$ isopropyl alcohol, 95\% isopropyl alcohol, cloth lubricant, mixture of $70 \%$ isopropyl alcohol with single iodopovidone droplet, and DMSO are shown in "Fig. 7. f.", "Fig. 7. g.", "Fig. 7. 
h.", "Fig. 7. i.", and "Fig. 7. j.", respectively. In this group of liquid samples, $70 \%$ isopropyl alcohol has highest yellow color spectrum than $95 \%$ isopropyl alcohol, cloth

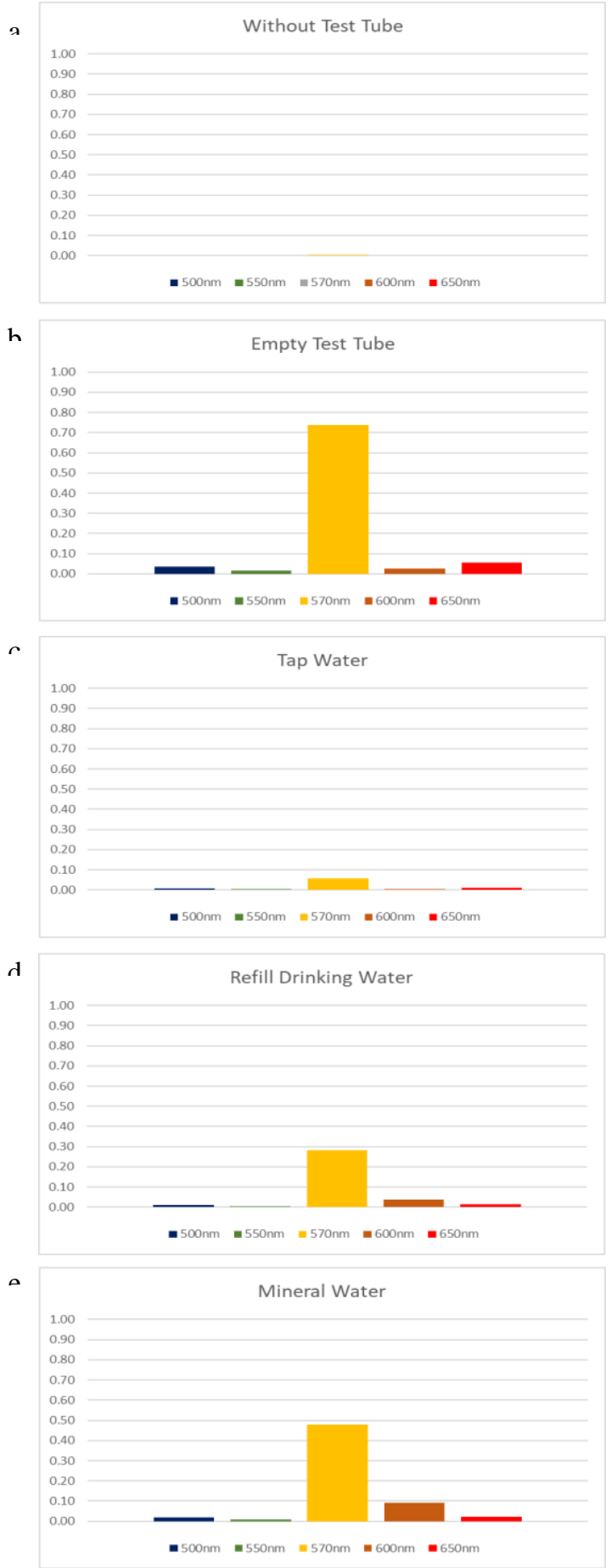

lubricant, mixture of $70 \%$ isopropyl alcohol and single droplet of iodopovidone, and dimethyl sulfoxide (DMSO).

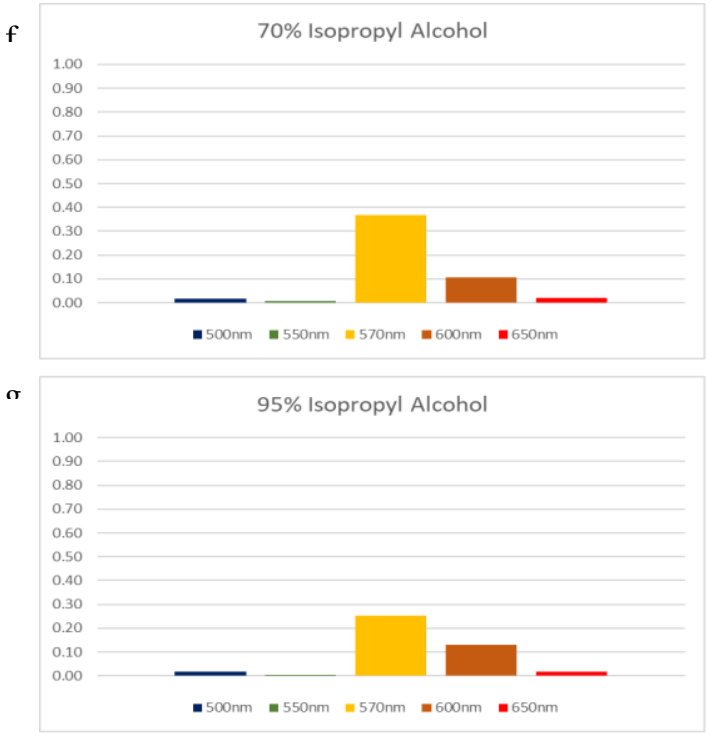

h
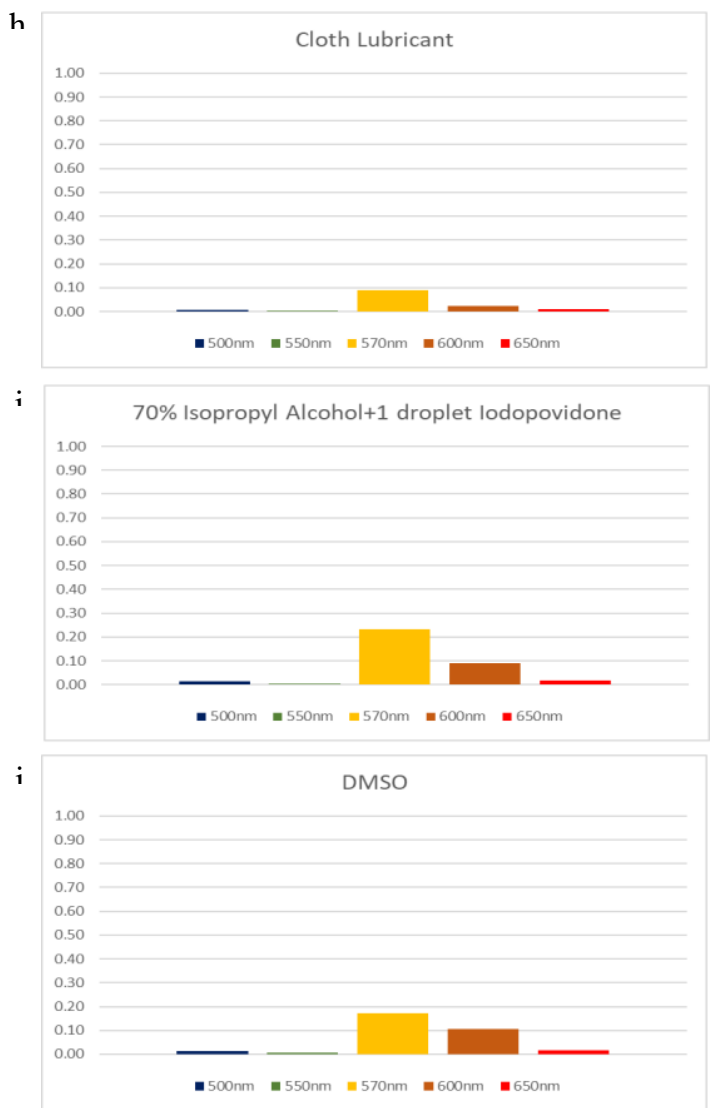

Fig. 7. Graph for normalized measurement results of Raman spectroscopy from each liquid samples and different measurement conditions

For Raman spectroscopy graphs that shown by "Fig. 7. a." until "Fig. 7. j.", the dominant light colors that received by the detector are yellow that has wavelength of 570nm. The detector also receives other light color spectrum that formed from scattering of violet-blue light from laser by liquid sample inside test tube, such as blue, green, orange, and red although not as strong as yellow light in appearance. This is byproduct of color shifting in Raman scattering. From this experiment, we acknowledge, even the liquid samples that we use to test our developed instrumentation are same transparent liquid but with different composition, they still have different Raman spectra. 


\section{CONCLUSION}

All hardware and component that we used for low cost optical-electronic sensor based on Raman spectroscopy has been tested. Combination between violet-blue laser diode, test tube, collecting lens, CMOS photodetector array as detector, and microcontroller are providing measurement result to liquid sample. Instead of using the whole Raman spectrum, there are five channels of wavelength we used, $500 \mathrm{~nm}, 550 \mathrm{~nm}, 570 \mathrm{~nm}, 600 \mathrm{~nm}$, and $650 \mathrm{~nm}$.

The developed instrumentation has been used for testing transparent liquid such as tap water, refill drinking water, mineral water, $70 \%$ isopropyl alcohol, $95 \%$ isopropyl alcohol, cloth lubricant, mixture of $70 \%$ isopropyl alcohol and single droplet of iodopovidone, and dimethyl sulfoxide (DMSO). From this experiment, even all liquid samples that used to test this combination of hardware and components are transparent, they still have different Raman spectra. For future work, this developed instrumentation can be applied into some further implementation for instance body liquid measurement such as blood. In specific application, we need to employ data analysis and neural network method combined with this developed instrumentation.

\section{REFERENCES}

[1] Smith, Ewen and Dent, Geoffrey, "Modern Raman Spectroscopy A Practical Approach," Chichester: John Wiley \& Sons Ltd, 2005.

[2] Tkachenko, Nikolai V., "Optical Spectroscopy - Methods and Instrumentations," Oxford: Elsevier B.V., 2006.

[3] Jun, Jiang, et al., "Simultaneous detection of dissolved methane and ethane in transformer oil based on laser Raman spectroscopy," 5th International Conference on Power Generation Systems and Renewable Energy Technologies (PGSRET), 26-27 August 2019.

[4] Arrobas, B.Gordillo, et al., "Raman spectroscopy for analyzing anthocyanins of lyophilized blueberries," 2015 IEEE Sensors, 2015.

[5] Zheng, Xiangxiang, et al., "Rapid and low-cost detection of thyroid dysfunction using Raman spectroscopy and an improved support vector machine," IEEE Photonics Journal, Vol. 10, No.6, 2018.

[6] Telle, Helmut H., Ureña, Angel González. and Donovan, Robert J., "Laser Chemistry - Spectroscopy, Dynamics, and Application," Chichester: John Wiley \& Sons Ltd, 2007.

[7] R. Kumar, Challa S. S. (ed), "Raman Spectroscopy for Nanomaterials Characterization," Heidelberg: Springer-Verlag Berlin Heidelberg, 2012.

[8] Scoicaru, L. O., et al., "New advances in analytic and diagnostic technologies based on Raman spectroscopy," ICTON 2017, 2017.

[9] Zezell, Denise Maria, et al., "Characterization of natural carious lesions by fluorescence spectroscopy at 405-nm excitation wavelength," Journal of Biomedical Optics, vol. 12(6), 2007.

[10] da Silva, Michael, et al., "Design and implementation of low cost optical spectrometer," Proceeding of the 2nd International Conference on Inventive Communication and Computational Technologies, pp. 1904-1908.

[11] Magnusson, R., "Light sources and optics," Encyclopedia of Spectrosopy and Spectrometry, vol.1, pp. 1158-1168, 1999.

[12] Ball, David W., "The Basics of Spectroscopy," Washington: The Society of Photo-Optical Instrumentation Engineers, 2001.

[13] Garbuny, Max., "Optical Physics," New York: Academic Press Inc, 1965.

[14] Gauglitz, Günter and Vo-Dinh, Tuan, "Handbook of Spectroscopy," Weinheim: WILEY-VCH Verlag GmbH \& Co. KGaA, 2003.

[15] Andrews, David L., "Rayleigh scattering and Raman effect, theory," Encyclopedia of Spectrosopy and Spectrometry, vol.1, pp. 19932000, 1999.

[16] Wartewig, Siegfried, "IR and Raman Spectroscopy," Weinheim: WILEY-VCH Verlag GmbH \& Co. KGaA, 2003.
[17] Dé Coster, Diane, et al., "Free-form optics enhanced confocal Raman spectroscopy for optofluidic lab-on-chips," IEEE Journal of Selected Topics in Quantum Electronics, vol. 21, 2015.

[18] McCreery, Richard L., "Raman Spectroscopy for Chemical Analysis," Canada: John Wiley \& Sons, Inc., 2000.

[19] Telle, Helmut H., Ureña, Angel González, and Donovan, Robert J., "Laser Chemistry - Spectroscopy, Dynamics, and Applications," Chichester: John Wiley \& Sons, Ltd, 2007.

[20] QPhotonics, "Single mode laser diode, 100mW @ 405nm, QLD405-100S," QLD-405-100 datasheet.

[21] Kumbhar, Kalpana and Kshirasagar, Ketki P., "Comparative study of CCD \& CMOS sensors for image processing," International Journal of Innovative Research in Electrical, Electronics, Instrumentation and Control Engineering, Vol. 3, pp. 194-196, December 2015.

[22] Blanc, Nicolas, "CCD versus CMOS - has CCD imaging come to an end?," Photogrammetric Week 01, pp. 131-137, 2003.

[23] ams, “AS7262 6-Channel Visible Spectral_ID Device with Electronic Shutter and Smart Interface," ams Datasheet, 17 March 2017. 